\title{
MIMO-Radar Waveform Design for Beampattern Using Particle-Swarm-Optimisation
}

\author{
Sajid Ahmed ${ }^{\dagger}$, John S. Thompson ${ }^{\ddagger}$ and Bernard Mulgrew ${ }^{\ddagger}$ \\ $\dagger$ King Abdullah University of Science and Technology, Thuwal, Makkah Province, Saudi Arabia. \\ Email: sajid.ahmed@kaust.edu.sa. \\ $\ddagger$ Institute for Digital Communications, School of Engineering, University of Edinburgh, EH8 3JL, UK \\ Email: john.thompson,b.mulgrew@ed.ac.uk
}

\begin{abstract}
Multiple input multiple output (MIMO) radars have many advantages over their phased-array counterparts: improved spatial resolution; better parametric identifiably and greater flexibility to acheive the desired transmit beampattern. The desired transmit beampatterns using MIMO-radar requires the waveforms to have arbitrary auto- and cross-correlations. To design such waveforms, generally a waveform covariance matrix, $R$, is synthesised first then the actual waveforms are designed. Synthesis of the covariance matrix, $R$, is a constrained optimisation problem, which requires $R$ to be positive semidefinite and all of its diagonal elements to be equal. To simplify the first constraint the covariance matrix is synthesised indirectly from its square-root matrix $U$, while for the second constraint the elements of the $m$-th column of $\mathrm{U}$ are parameterised using the coordinates of the $m$-hypersphere. This implicitly fulfils both of the constraints and enables us to write the cost-function in closed form. Then the cost-function is optimised using a simple particle-swarm-optimisation (PSO) technique, which requires only the cost-function and can optimise any choice of norm cost-function.
\end{abstract}

Index Terms-MIMO radar, colocated antennas, beampattern design, particle-swarm-optimisation.

\section{INTRODUCTION}

In contrast to phased-array radars, colocated multiple input multiple output (MIMO) radars allow each transmitting antenna to transmit independent waveforms and thus provide extra degrees-of-freedom (DOF) that can be exploited to improve system performance [1], [2]. These extra DOF can be utilised to design a wider range of beampattern. The design of waveforms for the desired beampattern, using colocated MIMOradar, generally requires the synthesis of the covariance matrix $\mathbf{R}$, which is a constrained optimization problem. Since $\mathbf{R}$ is a covariance matrix, the first constraint requires that it should be positive semidefinite. While for the ease of design and maximum power efficiency the second constraint requires that all of its diagonal elements, which represent the transmitted power from the corresponding antennas, must be equal.

The waveform design methods for colocated-radars to achieve a desired beampattern are discussed in [3]-[5]. These algorithms are discussed in detail in our previous work in [6]. The drawback with these algorithms is that they are all restricted to quadratic cost-functions. The algorithm in [3] has a lower computational complexity but violates the equal diagonal constraint to write the cost-function in closed-form. The performance of the algorithm in [4] due to the high dimensional search space and suboptimal scaling factor is poor.
The algorithm in [5] uses semidefinite-quadratic-programming (SQP) to solve the constrained optimisation problem, which is restricted to quadratic cost-functions only.

In [6], we have introduced a new square-root factorisation of the covariance matrix, which simultaneously guarantees that the matrix is positive semidefinite and has equal diagonal elements. In this algorithm, to fulfil the positive-semi-definite constraint, the matrix $\mathbf{R}$ is synthesised indirectly from an uppertriangular square-root matrix $\mathbf{U}$, such that $\mathbf{R}=\mathbf{U}^{H} \mathbf{U}$. To ensure that $\mathbf{R}$ has equal diagonal elements the $m$ th column of $\mathbf{U}$ is parameterised using the $m$-hyperspherical co-ordinate system of constant radius $r$. Thus, the square-root matrix and the covariance matrix itself are functions of the sines and cosines of spherical angles. These spherical angles can be optimised freely to synthesize a covariance matrix that approximates the desired beampattern and is guaranteed to be positive semidefinite and have equal diagonal elements. Having removed the constraints from the optimization problem, the need to use SQP methods is removed.

Since the sine and cosine functions are used to parametrise the covariance matrix, $\mathbf{R}$, the problem becomes non-linear. In [6], it is not proved that this non-linear problem is convex, however, gradient-descent and Newton-gradient algorithms are used to synthesise the covariance matrix $\mathbf{R}$ for the desired beampattern. These algorithms have showed no sensitivity to the initial values and for every set of initial values they converged to the same point. For such problems, where the convexity of the problem has a question mark, particle-swarm-optimisation (PSO) can be used [7]. Therefore, in this paper PSO is used to synthesise the covariance matrix, $\mathbf{R}$, for the desired beampattern using our proposed new square-root factorisation of the covariance matrix. The other motive to use PSO is the fact that it only requires the cost-function and does not require any differentiation or matrix inversion unlike gradient-descent and Newton gradient algorithm. Different cost-functions yield different beampatterns and PSO can be very easily used to synthesise the covariance matrix, $\mathbf{R}$, for any norm cost-function.

The paper is organised as follows. In the following section the problem is formulated. The parametrisation of covariancematrix is reviewed in Section III. The PSO for the problem is developed in section IV. Simulation results are given in Section VI, followed by our conclusions in Section VII.

Notation: Bold upper case letters, $\mathbf{X}$, and lower case letters, 
$\mathbf{x}$, respectively denote matrices and vectors. Transposition and conjugate transposition of a matrix are respectively denoted by $(.)^{T}$ and $(.)^{H}$. $\mathrm{E}\{$.$\} denotes the statistical expectation. The$ $(m, n)$ th element of a matrix $\mathbf{X}$ is denoted by $X_{m n}$ and $m$ th column vector of a matrix $\mathbf{X}$ is denoted by $\mathbf{x}_{m}$. Finally, the element wise multiplication is denoted by $\odot$.

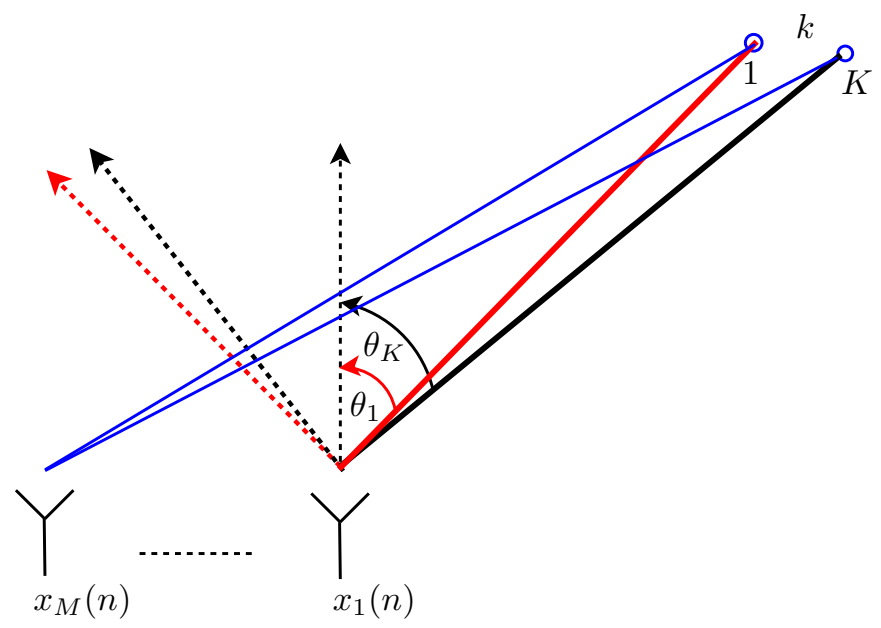

Fig. 1. An array of $M$ colocated transmit antennas, each one transmitting independent signals. The region of interest is divided into $K$ equally spaced angular locations.

\section{Problem Formulation}

The problem considered in this paper is to maximise the transmitted power at a number of given target locations and minimise it in all other locations. This is also called beampattern matching. To achieve this a uniform linear array of $M$ antenna elements with half-wavelength inter-element spacing, as shown in Fig.1, is used. Consider $x_{m}(n)$ to be the baseband transmitted signal from antenna $m$ at time index $n$ and $\theta_{k}$ is the spatial location. The baseband received signal at location $\theta_{k}$ can be written as

$r_{k}(n)=\sum_{m=1}^{M} e^{-j(m-1) \pi \sin \left(\theta_{k}\right)} x_{m}(n), \quad n=1,2, \ldots, N$.

By defining $\mathbf{e}\left(\theta_{k}\right)=\left[\begin{array}{llll}1 & e^{j \pi \sin \left(\theta_{k}\right)} & \cdots & e^{j(M-1) \pi \sin \left(\theta_{k}\right)}\end{array}\right]^{T}$ and $\mathbf{x}(n)=\left[\begin{array}{llll}x_{1}(n) & x_{2}(n) & \cdots & x_{M}(n)\end{array}\right]^{T}$ the received signal in (1) can be written as

$$
r_{k}(n)=\mathbf{e}^{H}(\theta) \mathbf{x}(n) .
$$

Following (2), the power received at location $\theta_{k}$ can be written as

$$
\begin{aligned}
P\left(\theta_{k}\right) & =\mathrm{E}\left\{\mathbf{e}^{H}\left(\theta_{k}\right) \mathbf{x}(n) \mathbf{x}^{H}(n) \mathbf{e}\left(\theta_{k}\right)\right\} \\
& =\mathbf{e}^{H}\left(\theta_{k}\right) \operatorname{Re}\left(\theta_{k}\right) .
\end{aligned}
$$

In order to achieve the desired beampattern an appropriate covariance matrix $\mathbf{R}$ has to be found. The matrix $\mathbf{R}$ can be optimised by minimising the difference between the desired and designed values of the beampattern. Therefore, to optimise $\mathbf{R}$ for the desired beampattern one cost-function can be defined as

$$
J(\Theta)=k_{1} \sum_{k=1}^{K}\left|\mathbf{e}^{H}\left(\theta_{k}\right) \operatorname{Re}\left(\theta_{k}\right)-\alpha \phi\left(\theta_{k}\right)\right|^{2},
$$

where $\alpha$ is the scaling factor and $\phi\left(\theta_{k}\right)$ is the desired beampattern. Since the matrix $\mathbf{R}$ is a covariance matrix of the waveforms, it must be positive semidefinite. Moreover, if all the antenna elements are required to transmit at the same maximum power level, all diagonal elements of the matrix $\mathbf{R}$ must be the same. Therefore, the matrix $\mathbf{R}$ cannot be chosen freely and has to satisfy the following constraints

$$
\begin{array}{ll}
\mathbf{C}_{1} . & \mathbf{R} \geq 0, \\
\mathbf{C}_{2} . & \mathbf{R}(m, m)=c, \text { for } m=1,2, \ldots, M,
\end{array}
$$

where $c$ is the transmitted power from each antenna. The first constraint can be achieved by constructing $\mathbf{R}$ with the matrix U such that

$$
\mathbf{R}=\mathbf{U}^{H} \mathbf{U}
$$

where $\mathbf{U}$ is a square-root of $\mathbf{R}$ and it can be an upper triangular (Cholesky factor) or a non-triangular matrix [8]. In order to achieve the second constraint the norm of the each column of $\mathrm{U}$ must be equal to $c$.

The motivation of our proposed work is to provide an unconstrained, simple and fast algorithm to synthesize the waveform covariance matrix. The proposed algorithm is an iterative algorithm that does not need any matrix factorisation or inversion and can be easily used even for non-quadratic cost-functions.

\section{Covariance Matrix Synthesis With PARAMETRISATION}

In this section new factorisation of the covariance matrix, which is described in our previous work, is reviewed. To fulfil the constraint $\mathbf{C}_{1}$, the covariance matrix $\mathbf{R}$ is synthesised indirectly from its square-root matrix $\mathbf{U}$. The square-root matrix $\mathbf{U}$ is an upper triangular matrix but is not necessarily a Cholesky factor of $\mathbf{R}$, i.e., it may have negative diagonal elements. To fulfil the constraint $\mathbf{C}_{2}$, the column vectors $\left\{\mathbf{u}_{i}\right\}_{i=1}^{M}$ of this square root matrix are parameterised using a multi-dimensional spherical co-ordinate system. The coordinates of any points on a multi-dimensional sphere have by definition a constant norm or radius $r$. Thus if spherical coordinates (with constant radius) are used to parametrise the columns of the square root matrix $\mathbf{U}$, constraints $\mathbf{C}_{1}$ and $\mathbf{C}_{2}$ are fulfilled and the spherical angles can be optimised freely to achieve the desired beampattern.

For example, the only non-zero element of the first column vector $\mathbf{u}_{1}$ can be parameterised by the coordinate of a single point at a distance $r$ from the origin as

$$
U_{11}=r .
$$

The first two non-zero elements of the second column vector $\mathbf{u}_{2}$ can be parameterised by the coordinates of a circle of radius $r$ 


$$
\mathbf{U}(\boldsymbol{\psi})=\left[\begin{array}{ccccc}
1 & \sin \left(\psi_{21}\right) & \sin \left(\psi_{31}\right) \sin \left(\psi_{32}\right) & \cdots & \prod_{m=1}^{M-1} \sin \left(\psi_{M m}\right) \\
0 & \cos \left(\psi_{21}\right) & \sin \left(\psi_{31}\right) \cos \left(\psi_{32}\right) & \cdots & \prod_{m=1}^{M-2} \sin \left(\psi_{M m}\right) \cos \left(\psi_{M, M-1}\right) \\
0 & 0 & \cos \left(\psi_{31}\right) & \vdots & \vdots \\
\vdots & \vdots & 0 & & \sin \left(\psi_{M 1}\right) \cos \left(\psi_{M 2}\right) \\
0 & \cdots & \cdots & 0 & \cos \left(\psi_{M 1}\right)
\end{array}\right]
$$

in terms of spherical-coordinates as

$$
\begin{aligned}
& U_{12}=r \sin \left(\psi_{21}\right), \\
& U_{22}=r \cos \left(\psi_{21}\right),
\end{aligned}
$$

where $\psi_{21}$ is the angular location of the point on the circle. Similarly, the upper three non-zero elements of $\mathbf{u}_{3}$ can be parameterised by the coordinates of a sphere of radius $r$ in terms of spherical-coordinates as

$$
\begin{aligned}
& U_{13}=r \sin \left(\psi_{31}\right) \sin \left(\psi_{32}\right), \\
& U_{23}=r \sin \left(\psi_{31}\right) \cos \left(\psi_{32}\right), \\
& U_{33}=r \cos \left(\psi_{32}\right),
\end{aligned}
$$

where $\psi_{31}$ and $\psi_{32}$ are respectively the elevation and azimuth angles of a sphere. These results can be easily extended for the remaining column vectors of $\mathbf{U}$ by using the coordinates of higher-dimensional spheres in terms of sphericalcoordinates. An $M \times M$ upper-triangular matrix $\mathbf{U}$ can be easily generated for radius $r=1$ as given in (6) above, where $\boldsymbol{\psi}=\left[\begin{array}{lllll}\psi_{21} & \psi_{31} & \psi_{32} & \cdots & \psi_{M, M-1}\end{array}\right]^{T}$. It can be noted in (6) that $m$ th column of $\mathbf{U}$ contains $(m-1)$ free angles $\left\{\psi_{m 1}, \psi_{m 2}, \ldots, \psi_{m, m-1}\right\}$ and for any value of these angles the $m$ th column of $\mathbf{U}$ has unit norm. The benefits of constructing $\mathbf{U}$ by this parametrisation are:

1) A simple gradient-descent algorithm can be used to find the elements of $\mathbf{U}$ without imposing any condition on the columns of $\mathbf{U}$ as imposed in [4].

2) The constraint $\mathbf{C}_{2}$ is guaranteed to be fulfilled, therefore the cost-function can be written in closed-form without any compromise on this constraint. The $\mathbf{C}_{2}$ constraint was violated in [3] to solve the cost-function in closedform.

3) Finally, in contrast to SQP in [5] the proposed algorithm can also be used for non-quadratic cost-functions.

The beampatterns are highly dependent on the cost-functions [9]. In the following two examples of a quadratic and a nonquadratic cost-function are given to synthesise the covariance matrix for the desired beampattern. Using the parameterised matrix $\mathbf{U}$ given in (6), the 1- and 2-norm un-constrained costfunctions can be written as

$$
\begin{aligned}
& J_{1}(\Theta)=k_{1} \sum_{k=1}^{K}\left|P\left(\boldsymbol{\psi}, \theta_{k}\right)-\alpha \phi\left(\theta_{k}\right)\right|, \\
& J_{2}(\Theta)=k_{1} \sum_{k=1}^{K}\left|P\left(\boldsymbol{\psi}, \theta_{k}\right)-\alpha \phi\left(\theta_{k}\right)\right|^{2},
\end{aligned}
$$

where $P\left(\boldsymbol{\psi}, \theta_{k}\right)=\mathbf{e}^{H}\left(\theta_{k}\right) \mathbf{U}^{H}(\boldsymbol{\psi}) \mathbf{U}(\boldsymbol{\psi}) \mathbf{e}\left(\theta_{k}\right)$ and $\Theta=$ $\left[\boldsymbol{\psi}^{T} \alpha\right]^{T} \in \Re^{L}$. The flexibility to choose freely the elements in the vector $\Theta$ without affecting the positive semidefinite and equal diagonal element properties of the matrix $\mathbf{R}$ is a significant advantage of this approach, which simplifies the application of iterative algorithms.

Since the geometric functions sine and cosine are used to parametrise the covariance matrix $\mathbf{R}$, the cost-functions in (7) and (8) are non-linear. Therefore, in the following section PSO is developed to synthesise the covariance-matrix $\mathbf{R}$.

\section{PARTICLE-SWARM-OPTIMISATION}

The details of the PSO can be found in [7] and the references therein. In PSO the variable to be found is called an agent or a particle. The coordinates of a particle, which represent the solution to the problem are called position of the particle. For this problem $L=\left(1+\sum_{m=1}^{M-1} m\right)$ variables need to be optimised, therefore the particle used for this problem is an $L$ dimensional vector. In PSO, initially $P$ particles each of dimension $L$ and their velocities are initialised with random numbers. Boundaries for particles and their velocities are defined and kept within the range throughout the algorithm. For each particle's position the value of the cost-function (fitting) is evaluated and the particle which best fits the cost-function is found. This particle is denoted by the vector $\mathrm{g}_{\text {best }}$ then the following iterative process is performed.

Step 1. In each iteration the velocity and position of each particle $i$ is updated as

$$
\begin{aligned}
\mathbf{v}_{i}= & w \mathbf{v}_{i}+c_{1} \operatorname{rand}(L) \odot\left(\Theta_{\text {best }}^{i}-\Theta_{\text {current }}^{i}\right) \\
& +c_{2} \operatorname{rand}(L) \odot\left(\mathbf{g}_{\text {best }}-\Theta_{\text {current }}^{i}\right), \\
\Theta_{i}= & \Theta_{i}+\mathbf{v}_{i},
\end{aligned}
$$

where rand is uniformly distributed pseudo random number, $\Theta_{\text {best }}^{i}$ is the best and $\Theta_{\text {current }}^{i}$ is the current position of the particle $i$. The letters $w, c_{1}$ and $c_{2}$ are constants, the value of $w$ is kept between 0 and 1 , while the values of $c_{1}$ and $c_{2}$ are kept close to 2 as explained in [7]. In the first iteration $\Theta_{\text {best }}^{i}$ is considered equal to the $\Theta_{\text {current }}^{i}$ for $i=1,2, \ldots, P$.

Step 2. Once the value of the particle $i$ is updated its fitness is evaluated. If the updated fitness of the particle is less then the previous best-fitness of the particle then $\Theta_{\text {best }}^{i}=\Theta_{\text {current }}^{i}$, similarly the best-fitness of the particle $i$ is considered equal to its current-fitness.

Step 3. The best-particle, $g_{\text {best }}$, whose fitness is the best fit of the cost-function is found.

Step 4. Go to the step-1 if the algorithm is not converged.

The steps in the PSO are summarised in the Table 1. 


\section{TABLE I}

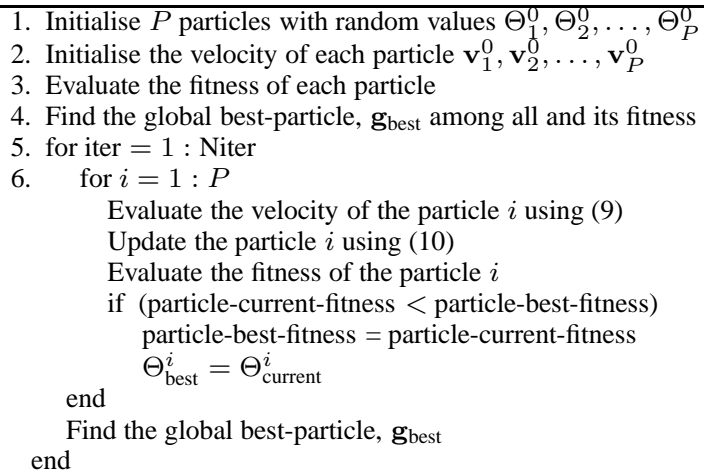

\section{WAVEFORM DESIGN}

Once the covariance matrix, $\mathbf{R}$, is synthesised the next step is to design the actual waveforms. The waveform matrix $\mathbf{X}=\left[\begin{array}{llll}\mathbf{x}_{1} & \mathbf{x}_{2} & \cdots & \mathbf{x}_{M}\end{array}\right]$ to realise a covariance matrix $\mathbf{R}$ can be determined as

$$
\begin{aligned}
\mathbf{X} & =\sqrt{N} \mathbf{P} \mathbf{R}^{1 / 2} \\
\text { or } \mathbf{X} & =\mathcal{X} \Lambda^{1 / 2} \mathbf{W}^{H},
\end{aligned}
$$

where $\mathbf{X} \in \mathcal{C}^{N \times M}, \mathbf{x}_{m}$ contains the symbols to be transmitted from antenna $m, \mathbf{P} \in \mathcal{C}^{N \times M}$ is a semi-orthonormal matrix, $\mathcal{X} \in \mathcal{C}^{N \times M}$ is a matrix of zero mean and unit variance Gaussian RV's, $\Lambda \in \mathcal{R}^{M \times M}$ is the diagonal matrix of eigenvalues and $\mathbf{W} \in \mathcal{C}^{M \times M}$ is a matrix of the eigenvectors of $\mathbf{R}$ [10]. The distribution of the RV's in the columns of $\mathbf{X}$ in (11) depends on $\mathbf{P}$, while the distribution in the columns of $\mathbf{X}$ in (12) is Gaussian. Neither equation (11) nor equation (12) guarantee a $\mathrm{CE}$ solution for the waveforms in $\mathbf{X}$.

By mapping Gaussian random-variables (RV's) onto BPSK RV's we have developed a relationship between crosscorrelation of Gaussian and BPSK RV's in [11]. This relationship is used to generate finite alphabet $\mathrm{CE}$ waveforms in [12]. Due to the limitation of space the waveform design is not considered further here.

\section{Simulation Results}

In this section, to validate the performance of the proposed algorithms some numerical examples are presented. In all of the following simulations a uniform linear array of 10 elements with a half-wavelength inter-element spacing is used and the transmitted power from each antenna is assumed to be equal to one. For all of the simulations in the following when using PSO, the constants $c_{1}=2, c_{2}=2$ and $w=1$ in (9) as suggested in most of the PSO algorithms.

In the first example, we consider the desired beampattern to be symmetric with a width of 60-degrees. For this beampattern, Stoica in [5] defines a 2-norm cost-function similar to (8) and uses SQP to synthesis the covariance matrix, $\mathbf{R}$. The same problem is solved using our proposed algorithm by incorporating PSO. Fig. 2 shows the desired and designed beampatterns using both of the algorithms, it can be seen in the figure that both algorithms approximate the desired beampattern identically.
The design of the beampattern depends strongly on the choice of cost-function. Therefore, in the second example, to compare the beampattern design using $1-, 1.5-$ and $2-$ norm cost-functions, the covariance matrix, $\mathbf{R}$, is synthesised using PSO for a beampattern in the previous example. Fig. 3 shows the corresponding beampatterns. It can be seen in the figure that the beampattern designed using a 1-norm costfunction performs better than the beampattern designed using $1.5-$ and $2-$ norm cost-functions. The former design transmits more power in the maximum power region, less power in the minimum power region and has significantly lower ripple amplitudes in both regions compared to the later ones.

To compare the convergence behaviour of PSO and gradientdescent algorithms the covariance matrix, $\mathbf{R}$, is synthesised to design a symmetric beampattern of width 60 degrees with PSO and gradient-descent algorithm using $1-$ and 2 -norm costfunctions. The step-size, $\mu$, for the gradient descent algorithm is 0.001 . Fig. 4 shows the mean and mean-square error between the desired and designed beampatterns for $1-$ and $2-$ norm cost-functions respectively. It can be seen in the figure that PSO not only has lower computational complexity but converges faster in approximately 200 iterations when compared with the gradient descent algorithm, which converges in approximately 1400 iterations. The other advantage of the 1 -norm costfunction, which can also be seen in the same figure, is that the convergence speed of the 1-norm cost-function is little bit faster than the 2-norm cost-function for both PSO and the gradientdescent algorithm.

In the last simulation, the requirement is to illuminate three targets at locations $-20,0$ and 20 degrees. PSO is used to synthesise the covariance matrix, $\mathbf{R}$, using $1-$ and $2-$ norm cost-functions to illuminate these targets. Fig. 5 shows the corresponding beampatterns. From these simulation results, it is clear that the 1-norm design better illuminates the targets compared to the 2 -norm design. Moreover, the 1 -norm design transmits less power in the unwanted region and its beampattern spatial resolution is much better compared to the 2-norm design. Therefore, it can be said that $1-$ norm is a better error criterion compared to the 2 -norm error criterion to design the beampattern, because it minimise the actual error between the desired and designed beampatterns instead of the squared error.

\section{CONCLUSIONS}

In the proposed algorithm the constrained optimization problem is converted into an unconstrained one by introducing and exploiting a new factorisation of the covariance matrix in terms of hyper-spherical co-ordinates. This new factorisation guarantees that the covariance matrix is both positive semidefinite and has equal diagonal elements. It also facilitates the use of the gradient-descent, conjugate-gradient and Newton-gradient techniques for the optimization of both quadratic and nonquadratic cost-functions. The possible non-convexity of the cost-functions, when parameterised in terms of hyper-spherical co-ordinates, is solved by using particle-swarm-optimisation. 


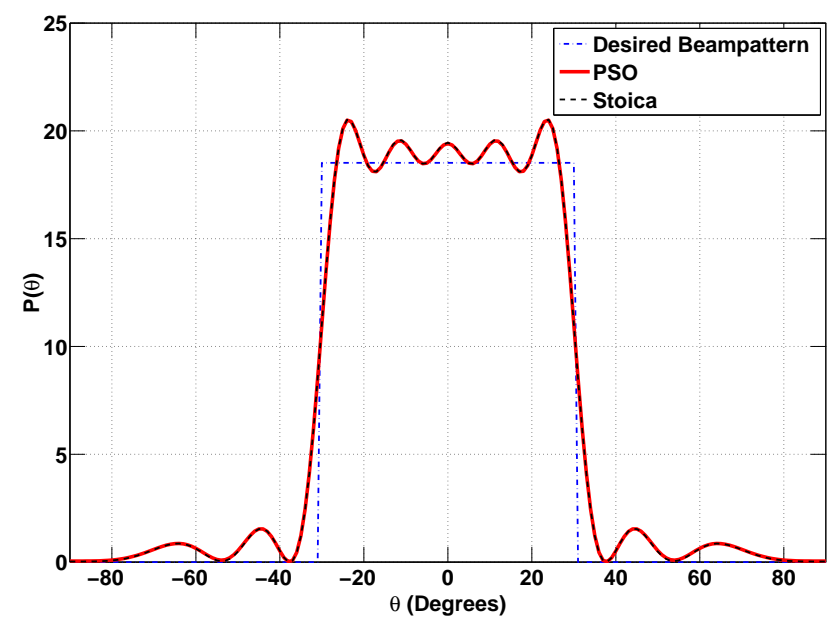

Fig. 2. Beampattern obtained using new square-root factorisation and PSO is compared with the beampattern using constrained algorithm proposed in [5].

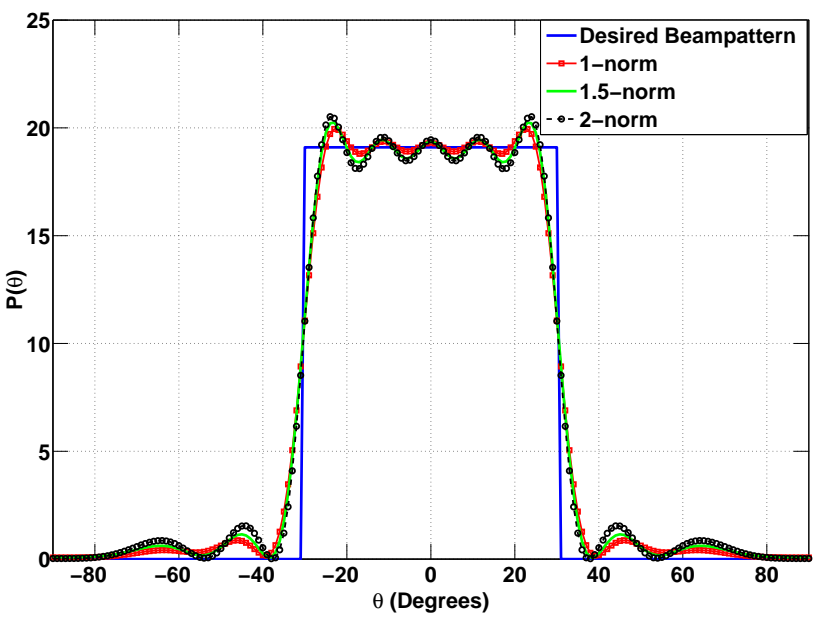

Fig. 3. Beampattern designed using $1-, 1.5-$, and $2-$ norm cost-functions Particle-swarm-optimisation is used to optimise the covariance matrix, $\mathbf{R}$, to obtain the desired beampattern.

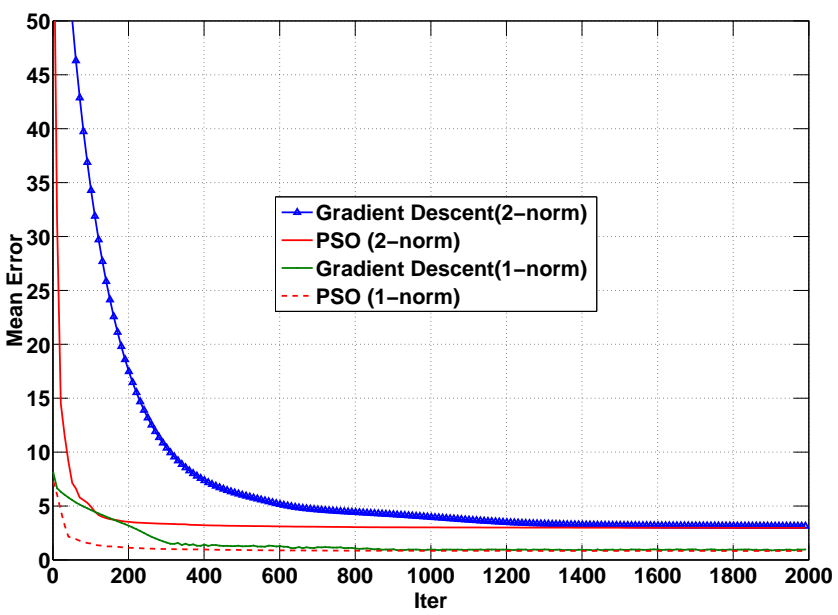

Fig. 4. Comparison of the convergence behaviour of gradient-descent algorithm with the convergence behaviour of PSO.

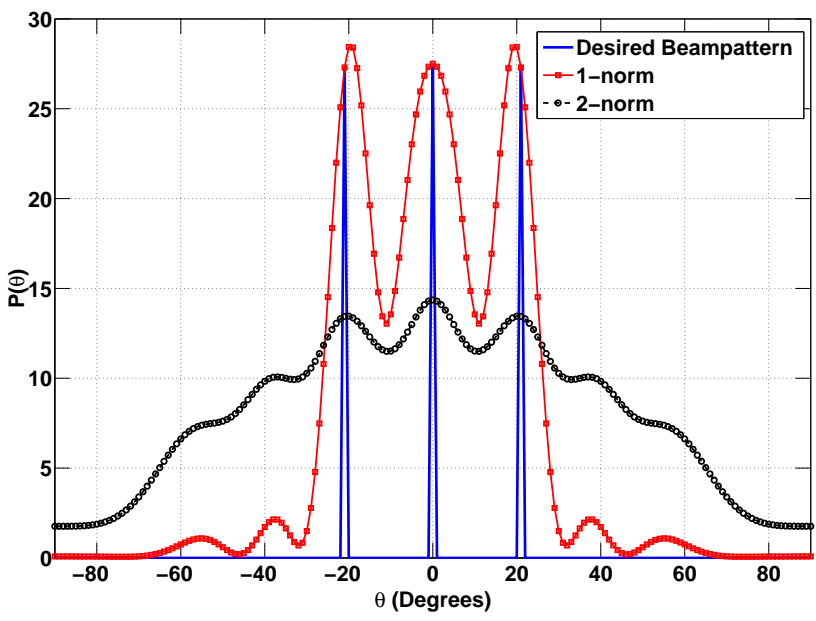

Fig. 5. The comparison of the obtained beampattern using $1-$ and 2 -norm cost-functions to illuminate three targets at locations $-20,0$ and 20 degrees.

Although the 1-norm error criterion is difficult to optimise compared to 2-norm error criterion, it can better approximate the desired beampattern. Since particle-swarm-optimisation only requires the cost-function, it can easily optimise the covariance matrix, $\mathbf{R}$, for the desired beampattern using the 1-norm costfunction.

\section{REFERENCES}

[1] E. Fishler, A. M. Haimovich, R. S. Blum, L. J. Cimini, D. Chizhik, and R. A. Valenzuela, "MIMO radar: An idea whose time has come," IEEE International Radar Conference, pp. 71-78, April 2004.

[2] E. Fishler, A. M. Haimovich, R. S. Blum, L. J. Cimini, D. Chizhik, and R. A. Valenzuela, "Spatial diversity in radars-models and detection performance," IEEE Transaction on Signal Processing, vol. 54, pp. 823838, Mar. 2006.

[3] T. Aittomaki and V. Koivunen, "Low complexity method for transmit beamforming in MIMO radar,' IEEE International Conference on Acoustics, Speech, and Signal Processing (ICASSP), pp. 305-308, Apr. 2007.

[4] D. R. Fuhrmann and J. S. Antonio, "Transmit beamforming for MIMO radar systems using signal cross-correlation," IEEE Transaction on Aerospace and Electronic Systems, vol. 44, pp. 171-185, Jan 2008.

[5] P. Stoica, J. Li, and Y. Xie, "On probing signal design for MIMO radar,' IEEE Transaction on Signal Processing, vol. 55, pp. 4151-4161, Aug. 2007.

[6] S. Ahmed, J. S. Thompson and B. Mulgrew, "Unconstrained synthesis of covariance matrix for MIMO radar transmit beampattern," IEEE Transaction on Signal Processing, vol. 59, no. 8, pp. 3837 - 3849, Aug. 2011.

[7] J. Robinson and Y. Rahmat-Samii, "Particle swarm optimization in electromagnetics, IEEE Transaction on Antenna and Propagation, vol. 52, no. 2, pp. 397-407, Feb. 2004

[8] G. H. Golub and C. F. V. Loan, Matrix computations. Johns Hopkins University Press, 3rd Ed., 1996.

[9] T. Aittomaki and V. Koivunen, "Signal covariance-matrix optimisation for transmit beamforming in MIMO radars," 41st Asilomar Conference on Signals, Systems and Computers, pp. 182-186, Nov. 2007.

[10] A. Hyvrinen, J. Karhunen, and E. Oja, Independent Component Analysis. John Wiley, 2001.

[11] S. Ahmed, J. S. Thompson, B. Mulgrew, and Y. Petillot, "Constant envelope waveform design for MIMO radar," IEEE International Conference on Acoustics Speech and Signal Processing (ICASSP), pp. 4066-4069, Mar. 2010.

[12] S. Ahmed, J. S. Thompson and B. Mulgrew, "Finite alphabet constantenvelope waveform design for MIMO radar beampattern," IEEE Transaction on Signal Processing, vol. 59, no. 11, pp. 5326 - 5337, Nov. 2011. 